\title{
FGFR4 Inhibitor FGF401
}

National Cancer Institute

\section{Source}

National Cancer Institute. FGFR4 Inhibitor FGF401. NCI Thesaurus. Code C120102.

An inhibitor of human fibroblast growth factor receptor 4 (FGFR4), with potential antineoplastic activity. Upon administration, FGF401 binds to and inhibits the activity of FGFR4, which leads to an inhibition of tumor cell proliferation in FGFR4-overexpressing cells. FGFR4 is a receptor tyrosine kinase upregulated in certain tumor cells and involved in tumor cell proliferation, differentiation, angiogenesis, and survival. 